\title{
Pulsar Glitches: The Crust may be Enough
}

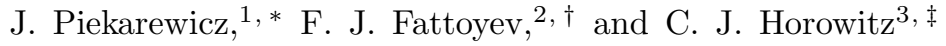 \\ ${ }^{1}$ Department of Physics, Florida State University, Tallahassee, FL 32306, USA \\ ${ }^{2}$ Department of Physics and Astronomy, Texas A $6 M$ University-Commerce, Commerce, TX 75429, USA \\ ${ }^{3}$ Center for Exploration of Energy and Matter and Department of Physics, \\ Indiana University, Bloomington, IN 47405, USA
}

(Dated: April 11, 2014)

\begin{abstract}
Background: Pulsar glitches - the sudden spin-up in the rotational frequency of a neutron star-suggest the existence of an angular-momentum reservoir confined to the inner crust of the neutron star. Large and regular glitches observed in the Vela pulsar have originally constrained the fraction of the stellar moment of inertia that must reside in the solid crust to about $1.4 \%$. However, crustal entrainment - which until very recently has been ignored - suggests that in order to account for the Vela glitches, the fraction of the moment of inertia residing in the crust must increase to about $7 \%$. This indicates that the required angular momentum reservoir may exceed that which is available in the crust.
\end{abstract}

Purpose: We explore the possibility that uncertainties in the equation of state provide enough flexibility for the construction of models that predict a large crustal thickness and consequently a large crustal moment of inertia.

Methods: Moments of inertia - both total and crustal - are computed in the slow-rotation approximation using a relativistic mean field formalism to generate the equation of state of neutron-star matter.

Results: We compute the fractional moment of inertia of neutron stars of various masses using a representative set of relativistic mean-field models. Given that analytic results suggest that the crustal moment of inertia is sensitive to the transition pressure at the crust-core interface, we tune the parameters of the model to maximize the transition pressure, while still providing an excellent description of nuclear observables. In this manner we are able to obtain fractional moments of inertia as large as $7 \%$ for neutron stars with masses below 1.6 solar masses.

Conclusions: We find that uncertainties in the equation of state of neutron-rich matter are large enough to accommodate theoretical models that predict large crustal moments of inertia. In particular, we find that if the neutron-skin thickness of ${ }^{208} \mathrm{~Pb}$ falls within the $(0.20-0.26) \mathrm{fm}$ range, large enough transition pressures can be generated to explain the large Vela glitches - without invoking an additional angular-momentum reservoir beyond that confined to the solid crust. Our results suggest that the crust may be enough.

PACS numbers: 97.60.Jd, 26.60.Kp, 26.60.Gj, 21.65.Ef

\footnotetext{
* jpiekarewicz@fsu.edu

$\uparrow$ farrooh.fattoyev@tamuc.edu

$\ddagger$ horowit@indiana.edu
} 


\section{INTRODUCTION}

Rotation-powered pulsars tend to spin down slowly and steadily due to the emission of magnetic dipole radiation, making pulsars one of nature's most accurate clocks. For example, the Crab pulsar-with a rotational period of $P \approx 33 \mathrm{~ms}$ - spins down at a rate of $\dot{P} / P \approx 1.3 \times 10^{-11} \mathrm{~s}^{-1}$ or about $13 \mu$ s per year. However, in spite of this remarkable regularity, young pulsars often display "glitches" which represent a sudden and abrupt spin-up in their rotational frequency. Through high-precision pulsar timing, an extensive glitch catalogue is now available which, at the time of this writing, has recorded a total of 439 glitches from 150 different pulsars 1, 2. Moreover, pulsar timing has revealed that glitches are recurrent - with some of the more active glitchers being the Vela with 17 , the Crab with 25 , and PSR B1737-30 with 33 [2].

The glitch mechanism is intimately related to the formation of superfluid vortices in the inner crust of the rotating neutron star [see Refs. 3, 4, and references contained therein]. Given that many pulsars rotate extremely fast, the areal density of superfluid vortices may be extremely high. For example, in the case of the Crab pulsar the vortex density reaches $n_{\mathrm{v}}=4 m_{n} / \hbar P \sim 2 \times 10^{5} \mathrm{~cm}^{-2}$, where $m_{n}$ is the neutron mass. The vortex density is so high that, although the bulk superfluid does not rotate, the superfluid as a whole appears to be rotating collectively as a "rigid" body [4. However, as the pulsar slows down by the emission of magnetic dipole radiation, the initial distribution of vortices - which are believed to be pinned to a crystal lattice of neutron-rich nuclei-falls out of equilibrium. This induces a differential rotation between the slower neutron star and the faster superfluid vortices. When the differential lag is too large, then suddenly and abruptly some fraction of the vortices unpin, migrate outwards, and transfer their angular momentum to the solid crust - and to any stellar component strongly coupled to it (such as the liquid core). This sudden (typically in less than a day) transfer of angular momentum is detected as a pulsar spin-up or a glitch. As a result of the glitch, the density of vortices diminishes from its pre-glitch value leaving the superfluid in close equilibrium with the solid crust. As the star continues to slow down over a period of days to years, stresses between the crust and the superfluid start to build up again until eventually more vortices unpin, transfer their angular momentum to the solid crust, and ultimately generate another glitch. The recurrence of glitches and the ensuing recovery is therefore a manifestation of a "sluggish" solid crust falling periodically out of equilibrium with the larger distribution of superfluid vortices.

Glitches from the Vela pulsar (B0833-45) were first observed in 1969 and have been continuously recorded for more than 40 years. So far a total of 17 glitches have been documented with individual spin-up rates of the order $\Delta \Omega / \Omega=10^{-6}$; note that although more glitches have been recorded for the Crab pulsar, the typical spin-up rate is three orders of magnitude smaller [1, 2, The high recurrence rate and large magnitude of the Vela glitches have been used to constrain the underlying equation of state (EOS) of neutron-star matter in terms of three measured quantities: its spin frequency $\Omega=70.338060018 \mathrm{~s}^{-1}$, its average spin-down rate $\dot{\Omega}=-9.8432 \times 10^{-11}$, and its glitch activity parameter $A_{g}$ - defined in terms of the cumulative spin-up rate $\sum_{n} \Delta \Omega_{n} / \Omega=2.9760 \times 10^{-5}$ as follows [5], 6 :

$$
A_{g}=\frac{1}{t} \sum_{n=1}^{N} \frac{\Delta \Omega_{n}}{\Omega} \approx 2.277 \times 10^{-14} \mathrm{~s}^{-1}
$$

where $t=41.421$ years is the total time elapsed between the first and the last $(N=17)$ glitches. Thus, in the standard model of pulsar glitches, the ratio of the moment of inertia of the superfluid component driving the glitches $I_{s}$ to the moment of inertia of the solid crust $I_{c}$ —plus any portion of the star strongly coupled to it - must satisfy the following inequality [5]:

$$
\frac{I_{s}}{I_{c}} \geq A_{g} \frac{\Omega}{|\dot{\Omega}|} \approx 0.016 .
$$

That is, long term timing observations of the Vela pulsar suggest that in order to account for its glitch activity, at least $1.6 \%$ of the stellar moment of inertia must reside in the superfluid reservoir [5 7]. Moreover, by adopting two plausible assumptions, one may rewrite the above limit in such a way as to provide a more meaningful constraint on the EOS. First, in the two-component model of Baym et al. [8], it is assumed that the component of the moment of inertia that rotates at the observed stellar frequency consists of the solid outer crust plus the liquid interior; then, $I_{c}=I-I_{s} \approx I$, where $I$ is the total stellar moment of inertia. Second, given that the inner crust is both thicker and denser than the outer crust, the crustal moment of inertia may be approximated as $I_{\text {crust }}=I_{\text {outer }}+I_{s} \approx I_{s}$. Thus, one may express the above constraint as follows:

$$
\frac{I_{s}}{I_{c}} \simeq \frac{I_{\text {crust }}}{I} \gtrsim 0.016 .
$$

This expression is particularly convenient as both $I_{\text {crust }}$ and $I$ may be readily evaluated in the slow-rotation approximation (see Sec II]. Indeed, most of the formalism relies on the solution of the Tolman-Oppenheimer-Volkoff equation 
whose only required input is the EOS of neutron-star matter. Further, whereas the total moment of inertia depends sensitively on the poorly constrained high-density component of the EOS, the crustal component $I_{\text {crust }}$ is sensitive to physics that may be probed in the laboratory. In fact, analytic expressions for $I_{\text {crust }}$ already exist that are both illuminating and highly accurate [see Refs. [5, 9, 10, and references contained therein]. In particular, the crustal moment of inertia is highly sensitive to physical observables - particularly the transition pressure - at the crust-core interface.

Recently, however, the standard glitch mechanism has been called into question [․ 11. It has been argued that crustal entrainment - the non-dissipative elastic scattering of unbound neutrons by the crystal lattice - effectively reduces the angular-momentum reservoir. That is, entrainment effects reduce the density of superfluid neutrons that could eventually become the source of the superfluid vortices. The impact from crustal entrainment may be encoded in an effective neutron mass $m_{n}^{\star} \equiv m_{n} n_{n}^{\mathrm{f}} / n_{n}^{\mathrm{c}}$, that reflects the ratio of unbound neutrons $n_{n}^{\mathrm{f}}$ (i.e., neutrons not bound to the nuclear lattice) to those $n_{n}^{\mathrm{c}}$ neutrons that are effectively free (i.e., not entrained) [6, 12]. As a result, crustal entrainment modifies the constraint given in Eq. (3) to

$$
\frac{I_{\text {crust }}}{I} \gtrsim 0.016\left(\frac{\left\langle m_{n}^{\star}\right\rangle}{m_{n}}\right) \simeq 0.07,
$$

where in the last expression we have adopted $\left\langle m_{n}^{\star}\right\rangle / m_{n} \simeq 4.3$ as suggested in Ref. [11]. Note that the amount of crustal entrainment may be uncertain, so more sophisticated calculations could yield a different constraint for the fractional moment of inertia. Here we assume, as in Ref. 11, that its value lies between the two limits given in Eqs. (3) and (4); that is, $0.016 \lesssim I_{\text {crust }} / I \lesssim 0.07$. Given the significant impact that crustal entrainment may have in constraining $I_{\text {crust }} / I$, it has been suggested that the crust is not enough, so that the core superfluid must also participate in glitches 11. However, before completely dismissing the standard glitch mechanism in favor of a more exotic explanation, we explore the conservative alternative that uncertainties in the EOS (which are large) could still allow the inner crust to account - by itself - for a large enough fraction of the moment of inertia to explain the large Vela glitches.

Uncertainties in the EOS are known to significantly affect the transition between the liquid interior and the solid crust. Particularly relevant to the present discussion is the poorly-known density dependence of the symmetry energy $S(\rho)$. The symmetry energy represents the energy required to convert symmetric nuclear matter into pure neutron matter at a fixed baryon density $\rho$. Although the symmetry energy at saturation density is fairly well determined, the value of its slope $L$ remains highly uncertain. And it is precisely the slope of the symmetry energy that controls the properties of neutron-rich matter at the crust-core transition region [13. Indeed, the transition density and proton fraction at the crust-core interface are both linearly anti-correlated to $L$. However, in stark contrast, the transition pressure does not vary monotonically with $L[10,14$. Note that such lack of correlation between the transition pressure and $L$-which appears to contradict some recent studies [15 19] - emerges only as one systematically explores a wide range of models. Given that the crustal moment of inertia grows with an increasing transition pressure, we want to explore the possibility of generating realistic EOS that predict large transition pressures. As we shall demonstrate, we find a class of relativistic mean field models with moderate values of $L$ that generate fractional moments of inertia as large as $I_{\text {crust }} / I \approx 0.09$ for a canonical $1.4 M_{\odot}$ neutron star. By using neutron star observations, Ref. [20] has recently and independently reached a similar conclusion. Moreover, we argue that these predictions may be directly tested in the laboratory. Although $L$ can not be directly measured in the laboratory, it is known to be strongly correlated to the neutron-skin thickness of ${ }^{208} \mathrm{~Pb}[21[24]$ - a fundamental nuclear-structure observable that has been measured [25, 26] and will be measured with increasing accuracy - at the Jefferson Laboratory [27. In summary, it is the main goal of the present work to explore whether present uncertainties in the EOS are large enough to accommodate realistic models that could account for large pulsar glitches - even in the presence of crustal entrainment.

We have organized the paper as follows. In Sec.[I] we review the essential details required to compute the stellar moment of inertia and the class of equations of state that will be used. In particular, special attention is paid to the crustal component of the moment of inertia and its sensitivity to the transition pressure at the crust-core interface. In Sec.III we provide predictions for the fraction of the moment of inertia residing in the crust. We show that although the transition pressure is sensitive to the slope of the symmetry energy, its behavior is not monotonic. This suggests a range of values for $L$ - that are neither too small nor too large - that provide the thickest crust and consequently the largest fractional moment of inertia. Finally, we offer our conclusions in Sec.IV]

\section{FORMALISM}

In this section we provide a brief review of the formalism required to compute the moment of inertia of a neutron star. First, we outline the procedure required to compute the stellar moment of inertia in the slow-rotation approximation. The enormous advantage of this approach is that most of what is required is a solution of the Tolman-OppenheimerVolkoff (TOV) equations in the limit of spherical symmetry. Given that the neutron-star matter equation of state is 
the sole ingredient required to solve the TOV equations, we devote a second subsection to review the main physical assumptions underlying our models.

\section{A. Moment of inertia of a neutron star}

In the slow-rotation approximation pioneered by Hartle and Thorne 28, 29] the moment of inertia of a uniformly rotating, axially symmetric neutron star is given by the following expression:

$$
I \equiv \frac{J}{\Omega}=\frac{8 \pi}{3} \int_{0}^{R} r^{4} e^{-\nu(r)} \frac{\bar{\omega}(r)}{\Omega} \frac{(\mathcal{E}(r)+P(r))}{\sqrt{1-2 G M(r) / r}} d r
$$

where $J$ and $\Omega$ are the angular momentum and rotational frequency of the neutron star. Further, $M(r), \mathcal{E}(r)$, and $P(r)$ are the stellar mass, energy density, and pressure profiles, respectively. Finally, $\nu(r)$ and $\omega(r)($ with $\bar{\omega}(r) \equiv \Omega-\omega(r))$ are radially-dependent metric functions. In the absence of general relativistic effects and in the simplified situation of a neutron star of uniform density, the stellar moment of inertia reduces to the elementary result of $I=2 M R^{2} / 5$. However, for realistic situations the stellar moment of inertia differs considerably from this elementary result. Note that in order for the slow-rotation approximation to be valid, the stellar frequency $\Omega$ must be far smaller than the mass-shedding Kepler frequency $\Omega_{\mathrm{K}}$. That is,

$$
\Omega \ll \Omega_{\mathrm{K}}=\sqrt{\frac{G M}{R^{3}}} \approx\left(1.15 \times 10^{4} \mathrm{~s}^{-1}\right) \sqrt{\frac{M / M_{\odot}}{\left(R / R_{10}\right)^{3}}},
$$

where in the last expression the stellar mass is expressed in solar masses and the radius in units of $R_{10} \equiv 10 \mathrm{~km}$. Equivalently, the slow-rotation approximation is valid if the period of the neutron star is significantly longer than about half a millisecond. Both the Crab and Vela pulsars - with periods of 33 and 89 milliseconds, respectively-fall safely within this bound.

The enormous virtue of the slow-rotation approximation is that every quantity that appears in Eq. 5 may be computed in the limit of spherical symmetry. As such, with the exception of the Lense-Thirring frequency $\omega(r)$, they may all be obtained from a solution of the Tolman-Oppenheimer-Volkoff (TOV) equations-properly supplemented by an equation of state $P=P(\mathcal{E})$. However, one should note that even under this simplified situation the formalism remains complex. Indeed, the Lense-Thirring precession is a qualitative new effect with no counterpart in Newtonian gravity that is caused by the dragging of inertial frames around a rotating compact star. Nevertheless, once the TOV equations are solved so that mass, energy density, and pressure profiles are obtained, the remaining quantities appearing in Eq. (5) may be computed by either performing a quadrature (in the case of $\nu$ ) or by solving a suitable differential equation (in the case of $\bar{\omega}$ ) [10]. The formalism for the moment of inertia of an axisymmetric star in hydrostatic equilibrium is derived in far greater detail in Refs. 30, 31, although a more pedagogical discussion may be found in the text by Glendenning $[32$.

Whereas the evaluation of the stellar moment of inertia relies on the numerical computation of various quantities, accurate and illuminating analytic expressions for the crustal moment of inertia already exist. The crustal component of the moment of inertia is defined in terms of the integral given in Eq. (5) but now with the integration range limited from the transition (or core) radius $R_{t}$ to the stellar radius $R$. That is,

$$
I_{\text {crust }}=\frac{8 \pi}{3} \int_{R_{t}}^{R} r^{4} e^{-\nu(r)} \widetilde{\omega}(r) \frac{(\mathcal{E}(r)+P(r))}{\sqrt{1-2 G M(r) / r}} d r
$$

However, given that the crust is thin and the density within it is low, a closed-form approximation for $I_{\text {crust }}$ has been obtained [5, 9, 10, 33, 35]. That is,

$$
I_{\text {crust }} \approx \frac{16 \pi}{3} \frac{R_{t}^{6} P_{t}}{R_{s}}\left[1-\frac{0.21}{\left(R / R_{s}-1\right)}\right]\left[1+\frac{48}{5}\left(R_{t} / R_{s}-1\right)\left(P_{t} / \mathcal{E}_{t}\right)+\ldots\right] .
$$

where $R_{s}=2 G M$ is the Schwarzschild radius of the star, and $P_{t}$ and $\mathcal{E}_{t}$ are the pressure and energy density at the crust-core interface. The ellipsis in the above equation indicates that the derivation was carried out to first order in the small (typically $\lesssim 1 \%$ ) quantity $P_{t} / \mathcal{E}_{t}$. Note that the TOV equation in the crustal region may be solved exactly using a polytropic EOS [10]. Although in reporting results for the crustal moment of inertia the exact expression given in Eq. (7) will always be used, the above analytic expression provides valuable insights. First, although approximate, 
the expression has been shown to be highly accurate 10. Second, the two terms that appear between brackets in Eq. (8) provide small $(\sim 10 \%)$ and canceling contributions to the leading term. Thus, the crustal moment of inertia of a neutron star of mass $M$ is dominated by $R_{t} \equiv R-R_{\text {crust }}$ and $P_{t}$ - quantities that are highly sensitive to the poorlyconstrained density dependence of the symmetry energy. Note that the low-density environment near the crust-core interface may be simulated in the collisions of heavy ions. Indeed, novel and unique experiments involving heavy-ion collisions with very neutron-rich nuclei at both present and future radioactive beam facilities will provide significant constraints on the EOS of neutron-rich matter [36].

\section{B. Equation of state for neutron-star matter}

Neutron stars are rich dynamical systems with core densities and neutron-proton asymmetries that far exceed those found in nuclei under normal conditions. As such, the EOS required to understand the structure and dynamics of neutron stars is highly uncertain and involves uncontrolled extrapolations. To mitigate this problem we rely on several nuclear-structure models that have been previously constrained by a variety of laboratory observables. These models, that fall under the rubric of relativistic mean-field models, provide a Lorentz covariant and causal framework that becomes essential as one extrapolates to the high densities encountered in the stellar core [37 [39].

The EOS for the uniform liquid core is based on an interacting Lagrangian density with its parameters accurately calibrated to a variety of nuclear properties. Besides nucleons, the uniform liquid core contains leptons - both electrons and muons - that enforce charge neutrality and chemical equilibrium and are treated as relativistic free Fermi gases. However, the model contains no exotic degrees of freedom. The interacting Lagrangian density includes a nucleon field $(\psi)$ interacting via the exchange of two isoscalar mesons (a scalar $\phi$ and a vector $V^{\mu}$ ) and one isovector meson $\left(b^{\mu}\right)$ [38, 39]. That is,

$$
\mathscr{L}_{\text {int }}=\bar{\psi}\left[g_{\mathrm{s}} \phi-\left(g_{\mathrm{v}} V_{\mu}+\frac{g_{\rho}}{2} \boldsymbol{\tau} \cdot \mathbf{b}_{\mu}\right) \gamma^{\mu}\right] \psi-U\left(\phi, V^{\mu}, \mathbf{b}^{\mu}\right),
$$

where $g_{\mathrm{s}}, g_{\mathrm{v}}$, and $g_{\rho}$ represent the Yukawa couplings between the nucleon and the corresponding meson fields. Although essential for the description of the properties of finite nuclei, note that the photon field does not contribute to the EOS at the mean-field level. In order to improve the phenomenological standing of the model, the Lagrangian density must be supplemented by nonlinear meson interactions that introduce an effective density dependence. These non-linear terms are given by

$$
U\left(\phi, V^{\mu}, \mathbf{b}^{\mu}\right)=\frac{\kappa}{3 !}\left(g_{\mathrm{s}} \phi\right)^{3}+\frac{\lambda}{4 !}\left(g_{\mathrm{s}} \phi\right)^{4}-\frac{\zeta}{4 !}\left(g_{\mathrm{v}}^{2} V_{\mu} V^{\mu}\right)^{2}-\Lambda_{\mathrm{v}}\left(g_{\rho}^{2} \mathbf{b}_{\mu} \cdot \mathbf{b}^{\mu}\right)\left(g_{\mathrm{v}}^{2} V_{\nu} V^{\nu}\right) .
$$

Historically, the first two terms $(\kappa$ and $\lambda)$ were introduced by Boguta and Bodmer to soften the compressibility coefficient of symmetric nuclear matter [40]. The last two terms $\left(\zeta\right.$ and $\left.\Lambda_{\mathrm{v}}\right)$ on the other hand, play a critical role in the behavior of the EOS at high densities. In particular, the isoscalar parameter $\zeta$ serves to soften the equation of state of symmetric nuclear matter at high densities 41 and, at present, can only be meaningfully constrained by the maximum mass of a neutron star 42, 43. Finally, the mixed isoscalar-isovector parameter $\Lambda_{\mathrm{v}}$ was introduced to modify the poorly-constrained density dependence of the symmetry energy [13, 44. Tuning this parameter provides a simple, efficient, and reliable method of softening the symmetry energy. Further details on the calibration procedure may be found in Refs. [13, 45, 46] and references contained therein.

As one moves away from the stellar core the density diminishes until the uniform system becomes unstable against cluster formation. That is, at densities of about one half of nuclear-matter saturation density, it becomes energetically favorable for the uniform system to cluster into neutron-rich fragments that are embedded in a dilute neutron vapor. Such instability delineates the transition between the uniform liquid core and the non-uniform solid crust. The stellar crust itself is divided into an inner and an outer region. In the outer crust the system is organized into a Coulomb lattice of neutron-rich nuclei embedded in a degenerate electron gas [47, 48. The composition in this region is solely determined by the masses of neutron-rich nuclei in the $26 \leq Z \lesssim 50$ region and the pressure support is provided by the degenerate electrons. For this region we adopt the standard equation of state of Baym, Pethick, and Sutherland (BPS) 47]. In contrast, the EOS for the inner crust is highly uncertain and must be inferred from theoretical calculations. In addition to a Coulomb lattice of progressively more exotic neutron-rich nuclei embedded in an electron gas, the inner crust now includes a dilute vapor of superfluid neutrons. Moreover, at the bottom layers of the inner crust, complex and exotic structures with almost equal energy — "nuclear pasta" - have been predicted to emerge [33, 49, 50]. Whereas significant progress has been made in simulating this exotic region [51 56], a detailed equation of state is still missing. Hence, we resort to a polytropic EOS to interpolate between the solid outer crust and the uniform liquid interior [5, 57. 
In summary, the equation of state adopted in this work is comprised of three parts: (i) a BPS component up to a neutron-drip density of $\rho_{\text {drip }} \approx 4 \times 10^{11} \mathrm{~g} / \mathrm{cm}^{3}$ to describe the outer crust; (ii) a polytropic component for the inner crust that interpolates between $\rho_{\text {drip }}$ and the crust-core transition density $\rho_{t}$; and (iii) a RMF component to describe the uniform liquid core. Note that $\rho_{t}$ is a model-dependent quantity highly sensitive to the density dependence of the symmetry energy. Consistency demands that the same RMF model used to generate the EOS will also be used to predict the crust-core transition density. In particular, the instability of the uniform ground state against cluster formation will be determined through a relativistic random phase approximation (RPA) as detailed in Refs. [13, 57.

\section{RESULTS}

In this section we display results for the fraction of the stellar moment of inertia residing in the non-uniform crust. By using a variety of realistic equations of state, we test the basic premise of our work, namely, whether the crust may be enough to explain large pulsar glitches. We start by displaying in Fig.1 the fraction of the crustal moment of inertia for a variety of neutron-star masses for two "families" of relativistic mean-field models: FSUGold [58] (or "FSU" for short) and NL3 59, 60. Note that each member of the family is characterized by a unique value of the neutron-skin thickness of ${ }^{208} \mathrm{~Pb}$. The original two models - with values of $R_{\text {skin }}^{208}=0.21 \mathrm{fm}$ for FSU and $R_{\text {skin }}^{208}=0.28 \mathrm{fm}$ for NL3 - were accurately calibrated to several nuclear properties and have been fairly successful in describing a variety of nuclear phenomena. Yet, from the perspective of the EOS, a critical difference between these two models is that NL3 predicts a significantly stiffer EOS than FSU. In particular, NL3 predicts a maximum neutron star mass almost one solar mass heavier than FSU. Moreover, with a symmetry energy also significantly stiffer, NL3 predicts considerably larger stellar radii. However, the density dependence of the symmetry energy may be efficiently modified by tuning the isoscalar-isovector coupling $\Lambda_{\mathrm{v}}$. Indeed, it is precisely through the tuning of $\Lambda_{\mathrm{v}}$ that we have generated the two families of RMF models displayed in Fig.1. Note that in doing so, all isoscalar properties - such as the EOS of symmetric nuclear matter - are left intact [10. Also note that we have quantified the sensitivity of the crustal fraction of the moment of inertia to the density dependence of the symmetry energy in terms of $R_{\text {skin }}^{208}$. This provides an attractive connection between a laboratory measurement and an astrophysical observation.
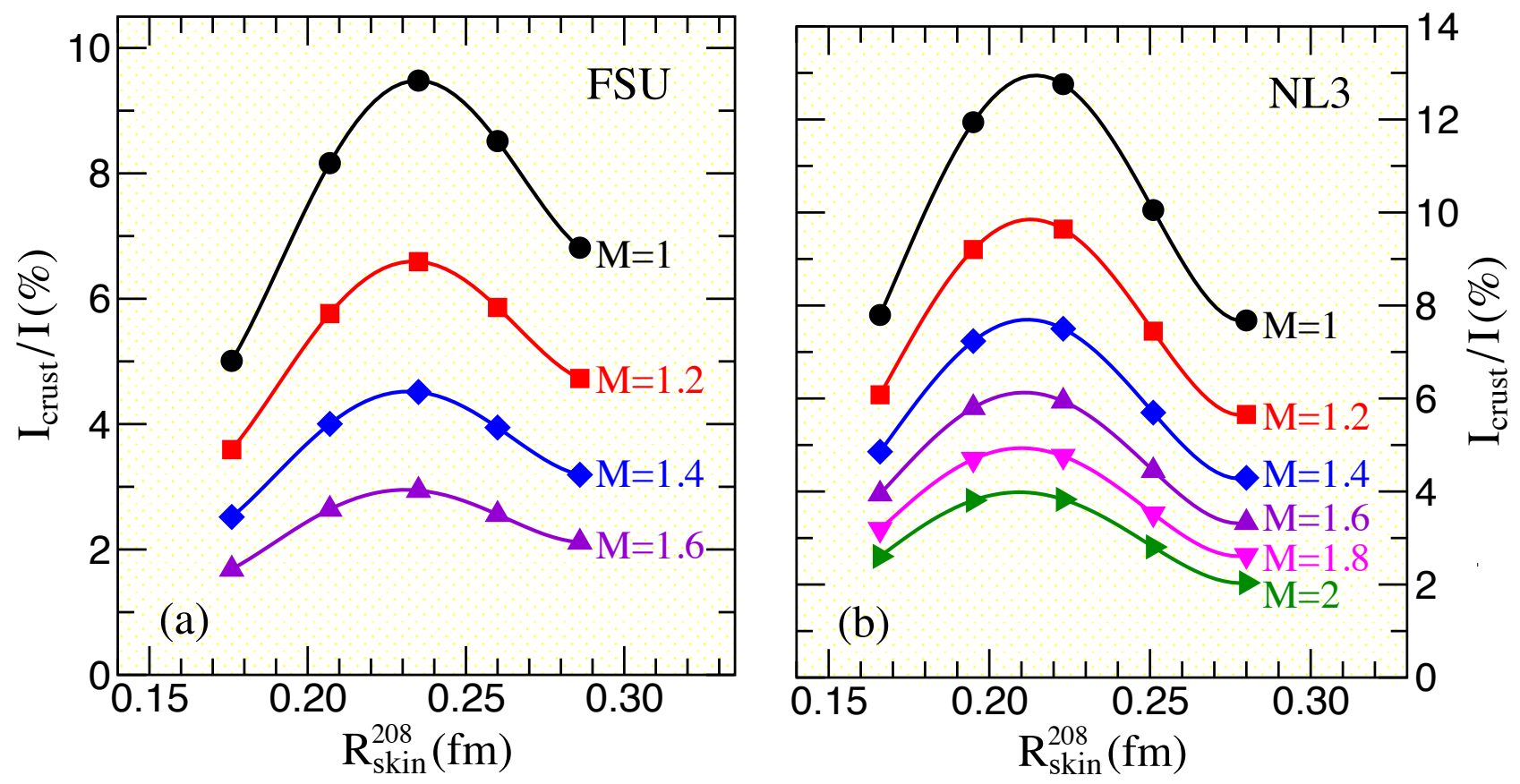

FIG. 1. (Color online) Fraction of the crustal moment of inertia as a function of the neutron-skin thickness of ${ }^{208} \mathrm{~Pb}$ for a variety of neutron-star masses (in units of the solar mass) as predicted by two "families" of mean-field models. (a) Predictions made with the relatively soft FSU [58] parametrization and (b) with the relatively stiff NL3 interaction [59, 60].

The lack of a linear correlation between the neutron skin thickness $R_{\text {skin }}^{208}$ and the fraction of the crustal moment of inertia is clearly evident in Fig.1. Whereas earlier studies have found a strong linear correlation between $R_{\text {skin }}^{208}$ 
and various properties of the EOS in the crust-core transition region 10, 13 - such as the baryon density, energy density, and proton fraction-no such correlation was observed in the case of the transition pressure [10, 14. Given the sensitivity of the crustal moment of inertia to the transition pressure, as indicated in Eq. (8), we now use such lack of correlation to identify the particular member of the FSU and NL3 families that yields the largest transition pressure and, consequently, the largest fraction of the crustal moment of inertia. As suggested by the results displayed in Fig.1, this occurs for models with "intermediate" values for the neutron-skin thickness of ${ }^{208} \mathrm{~Pb}$.

\begin{tabular}{|l||c|c|c|c|c|c|c|c|c|c|}
\hline Model & $m_{\mathrm{s}}$ & $m_{\mathrm{v}}$ & $m_{\rho}$ & $g_{\mathrm{s}}^{2}$ & $g_{\mathrm{v}}^{2}$ & $g_{\rho}^{2}$ & $\kappa$ & $\lambda$ & $\zeta$ & $\Lambda_{\mathrm{v}}$ \\
\hline IU-FSU & 491.500 & 782.500 & 763.000 & 99.4266 & 169.8349 & 184.6877 & 3.3808 & $+2.9600 \times 10^{-4}$ & 0.0300 & $4.6000 \times 10^{-2}$ \\
FSUmax & 491.500 & 782.500 & 763.000 & 112.1996 & 204.5469 & 122.9556 & 1.4203 & $+2.3762 \times 10^{-2}$ & 0.0600 & $2.4776 \times 10^{-2}$ \\
NL3max & 508.194 & 782.501 & 763.000 & 104.3871 & 165.5854 & 119.3049 & 3.8599 & $-1.5905 \times 10^{-2}$ & 0.0000 & $2.6708 \times 10^{-2}$ \\
TFcmax & 496.800 & 782.500 & 763.000 & 113.9565 & 198.0546 & 149.9692 & 2.6078 & $-1.8640 \times 10^{-3}$ & 0.0200 & $1.5133 \times 10^{-2}$ \\
\hline
\end{tabular}

TABLE I. Parameter sets for the relativistic mean-field models used in the text. The parameter $\kappa$ and the meson masses $m_{\mathrm{s}}$, $m_{\mathrm{v}}$, and $m_{\rho}$ are all given in $\mathrm{MeV}$. The nucleon mass has been fixed at $M=939 \mathrm{MeV}$ in all models.

The parameters for the four RMF models employed in this manuscript have been listed in TableI. We reiterate that all these models provide a fairly accurate description of various properties of finite nuclei throughout the nuclear chart. In particular, the IU-FSU parametrization was derived from the original FSUGold model by adjusting a few empirical parameters with the goal of softening the symmetry energy (in order to generate smaller stellar radii) and stiffening the overall EOS at higher density (to generate a larger limiting neutron-star mass) 61. In turn, the "FSUmax" set represents that particular member of the FSU family the predicts the maximum value for the transition pressure $P_{t}$. The remaining two sets, "NL3max" and "TFcmax", were selected in a similar fashion from the corresponding NL3 [59, 60] and TFc 46] families. That is, with the exception of IU-FSU that was selected as an example of a model with a fairly soft symmetry energy, the remaining three models were chosen to maximize the value of the transition pressure.

\begin{tabular}{|l||c|c|c|c|c|c||c|c|c|}
\hline Model & $\rho_{0}\left(\mathrm{fm}^{-3}\right)$ & $\varepsilon_{0}$ & $K_{0}$ & $J$ & $L$ & $K_{\text {sym }}$ & $B / A$ & $R_{\text {ch }}(\mathrm{fm})$ & $R_{\text {skin }}(\mathrm{fm})$ \\
\hline \hline IU-FSU & 0.155 & -16.40 & 231.33 & 31.30 & 47.20 & +28.53 & -7.89 & 5.48 & 0.16 \\
FSUmax & 0.148 & -16.30 & 230.01 & 33.23 & 66.00 & -65.59 & -7.89 & 5.51 & 0.22 \\
NL3max & 0.148 & -16.24 & 271.54 & 32.14 & 59.00 & -25.44 & -7.92 & 5.52 & 0.20 \\
TFcmax & 0.148 & -16.46 & 260.49 & 38.28 & 74.00 & -67.78 & -7.89 & 5.51 & 0.26 \\
\hline Experiment & & & & & & & -7.87 & 5.50 & $0.33_{-0.18}^{+0.16}$ \\
\hline
\end{tabular}

TABLE II. Predictions for the bulk parameters characterizing the behavior of infinite nuclear matter at saturation density $\rho_{0}$. The quantities $\varepsilon_{0}$ and $K_{0}$ represent the binding energy per nucleon and incompressibility coefficient of symmetric nuclear matter, whereas $J, L$, and $K_{\text {sym }}$ represent the energy, slope, and curvature of the symmetry energy. Also shown are the binding energy per nucleon, charge radius, and neutron-skin thickness of ${ }^{208} \mathrm{~Pb}$, alongside the corresponding experimental values. All quantities are given in $\mathrm{MeV}$ except otherwise indicated.

Before displaying our results for various crustal properties, we discuss briefly some of the uncertainties in the EOS of relevance to the present work. We start by listing in Table II model predictions for a few bulk parameters that characterize the behavior of asymmetric nuclear matter in the vicinity of saturation density. That is, we write the energy per nucleon as follows:

$$
\frac{E}{A}(\rho, \alpha)=\left(\varepsilon_{0}+\frac{1}{2} K_{0} x^{2}+\ldots\right)+\left(J+L x+\frac{1}{2} K_{\mathrm{sym}} x^{2}+\ldots\right) \alpha^{2}+\ldots
$$

where $\alpha \equiv(N-Z) / A$ is the neutron-proton asymmetry and $x \equiv\left(\rho-\rho_{0}\right) / 3 \rho_{0}$ quantifies the deviations of the density from its saturation value 62,63 . Moreover, $\varepsilon_{0}$ and $K_{0}$ represent the binding energy per nucleon and incompressibility coefficient of symmetric nuclear matter, whereas $J, L$, and $K_{\text {sym }}$ represent the energy, slope, and curvature of the symmetry energy. Also shown in TableII are predictions for the binding energy per nucleon, charge radius, and neutron-skin thickness of ${ }^{208} \mathrm{~Pb}$ - alongside the measured experimental values. Note that the pioneering Lead Radius Experiment (PREX) at the Jefferson Laboratory has recently provided the first electroweak determination of the neutron radius of ${ }^{208} \mathrm{~Pb}$. Given that its charge radius has been accurately known for many years, PREX effectively measured the neutron-skin thickness of ${ }^{208} \mathrm{~Pb}$ to be [25, 26]:

$$
R_{\text {skin }}^{208}=0.33_{-0.18}^{+0.16} \mathrm{fm}
$$




\begin{tabular}{|l||c|c|c|c||c|c|c|}
\hline Model & $\rho_{t}\left(\mathrm{fm}^{-3}\right)$ & $Y_{t}$ & $\mathcal{E}_{t}\left(\mathrm{MeV} \mathrm{fm}^{-3}\right)$ & $P_{t}\left(\mathrm{MeV} \mathrm{fm}^{-3}\right)$ & $M_{\text {crust }}\left(M_{\odot}\right)$ & $R_{\text {crust }}(\mathrm{km})$ & $I_{\text {crust }} / I(\%)$ \\
\hline \hline IU-FSU & 0.0871 & 0.0438 & 82.655 & 0.289 & 0.019 & 0.989 & 2.827 \\
FSUmax & 0.0727 & 0.0321 & 68.810 & 0.425 & 0.027 & 1.430 & 4.407 \\
NL3max & 0.0826 & 0.0357 & 78.290 & 0.550 & 0.052 & 1.990 & 7.619 \\
TFcmax & 0.0794 & 0.0484 & 75.454 & 0.692 & 0.061 & 2.372 & 9.258 \\
\hline
\end{tabular}

TABLE III. Predictions for the transition density, proton fraction, energy density, and pressure at the crust-core interface. Also shown are predictions for the crustal mass, radius, and fractional moment of inertia for a $1.4 M_{\odot}$ neutron star.

The results in Table II indicate that although the extensive experimental database of nuclear masses and charge radii is sufficient to constrain some of the bulk parameters of neutron-rich matter, it is insufficient to constrain them allparticularly those associated with the density dependence of the symmetry energy. It is precisely this flexibility that we have exploited in constructing theoretical models that predict large transition pressures without compromising their ability to describe well-measured nuclear observables.

To further elucidate some of the uncertainties in the EOS that are of relevance to the transition region between the liquid core and the solid crust, we list in Table $\Pi I I$ the density, proton fraction, energy density, and pressure at the crust-core interface. Also shown are predictions for the crustal mass, radius, and fractional moment inertia for a "canonical" $1.4 M_{\odot}$ neutron star. As expected, crustal properties are strongly correlated to the transition pressure $P_{t}$. Indeed, the larger the value of $P_{t}$, the larger the fraction of the mass, radius, and moment of inertia contained in the solid crust. To complement the tabular information we display in Fig.2 the EOS, i.e., pressure as a function of baryon density, for uniform neutron-star matter. Note that as a result of the saturation of symmetric nuclear matter, the pressure in the vicinity of saturation density $\left(\rho_{0} \simeq 0.15 \mathrm{fm}^{-3}\right)$ is dominated by the contribution from the symmetry energy; that is, $P \approx \rho_{0} L / 3$. Moreover, since $L$ is strongly correlated to $R_{\text {skin }}^{208}$, one can readily infer the relative size of the neutron-skin thickness of ${ }^{208} \mathrm{~Pb}$ from the value of the pressure at saturation density. Remarkably, this same pressure controls the stellar radius of low-mass neutron stars [57] (see Fig.57). However, whereas stellar radii are sensitive to the density dependence of the symmetry energy in the immediate vicinity of nuclear-matter saturation density [9], the maximum neutron-star mass depends on the high-density component of the EOS - which is largely controlled by the parameter $\zeta$ [see Eq. 100]. In particular, the smaller the value of $\zeta$ the larger the limiting neutron-star mass. Thus, by tuning $\zeta$ one can efficiently increase the maximum neutron-star mass without affecting the EOS at normal nuclear densities [41. Indeed, for the RMF models employed here one obtains: $M_{\max } / M_{\odot}=1.72,1.94,2.14$, and 2.75, for FSUmax, IU-FSU, TFcmax, and NL3max, respectively. Finally, the insert displays the EOS (on a linear scale) starting from the transition point up to saturation density. Indicated with the various symbols are the predictions from the different models for the crust-core transition density and pressure. Clearly, the behavior of $P_{t}$ is not monotonic; whereas TFcmax predicts the largest transition pressure, its transition density is neither the smallest nor the largest.

Having highlighted the most critical features of the EOS, we are now in a position to discuss our predictions for various crustal properties - specifically the mass, radius, and fractional moment of inertia. We start by displaying in Fig. 3 the crustal contribution to the mass and radius of neutron stars of various masses as predicted by the four RMF models considered in the text. For reference, we provide approximate analytic expressions for these two quantities. That is [10,

$$
\begin{aligned}
& M_{\text {crust }} \approx 8 \pi \frac{R_{t}^{4} P_{t}}{R_{s}}\left(1-R_{s} / R_{t}\right), \\
& R_{\text {crust }} \approx 8 \frac{R_{t}^{2} P_{t}}{R_{s} \mathcal{E}_{t}}\left(1-\frac{8 R_{t} P_{t}}{R_{s} \mathcal{E}_{t}}\right)^{-1} .
\end{aligned}
$$

As in the case of the crustal moment of inertia given in Eq. (8), both $M_{\text {crust }}$ and $R_{\text {crust }}$ are proportional to the product of the core radius $R_{t}$ (raised to some power) times the transition pressure $P_{t}[10$. Such a dependence is clearly displayed in the figure. We observe that whereas only a very small fraction of the total neutron-star mass resides in the low-density crust, the crustal contribution to the overall size of the star may be significant. For example, for the case of a $1.4 M_{\odot}$ neutron star, the TFcmax model predicts that of the total $14.4 \mathrm{~km}$ stellar radius, $2.4 \mathrm{~km}$ (or $\simeq 17 \%$ ) are accounted by the crust. Could such a thick crust account for the large pulsar glitches?

To answer this question we have computed the crustal fraction of the total moment of inertia as a function of the neutron-star mass. Our results are displayed in Fig. 4 alongside the observational constraints imposed from the large Vela glitches. Here we follow the assumptions of Ref. [1] that suggest that in order to explain the large Vela glitches $1.6 \%$ of the moment of inertia must reside in the superfluid reservoir-but that such number must be increased to almost $7 \%$ if entrainment effects (with $\left\langle m_{n}^{*}\right\rangle / m_{n}=4.3$ ) are included. As one may expect from the sensitivity of crustal properties to the transition pressure, the trends displayed in Fig. 4 follow closely those observed in Fig. 3 . 


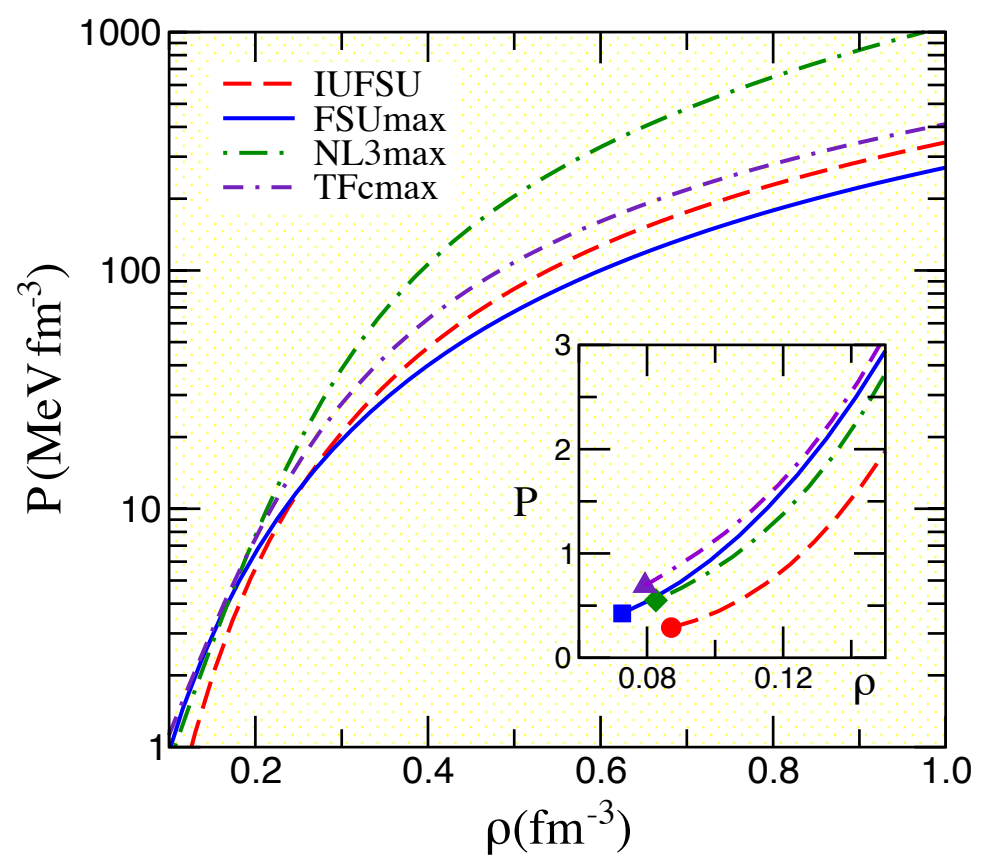

FIG. 2. (Color online) Equation of state in the form of pressure-vs-baryon density as predicted by the four models considered in the text. The insert displays (on a linear scale) the EOS on a more limited range: from the crust-core transition density up to nuclear-matter saturation density. The symbols denote the density and pressure at the crust-core interface.
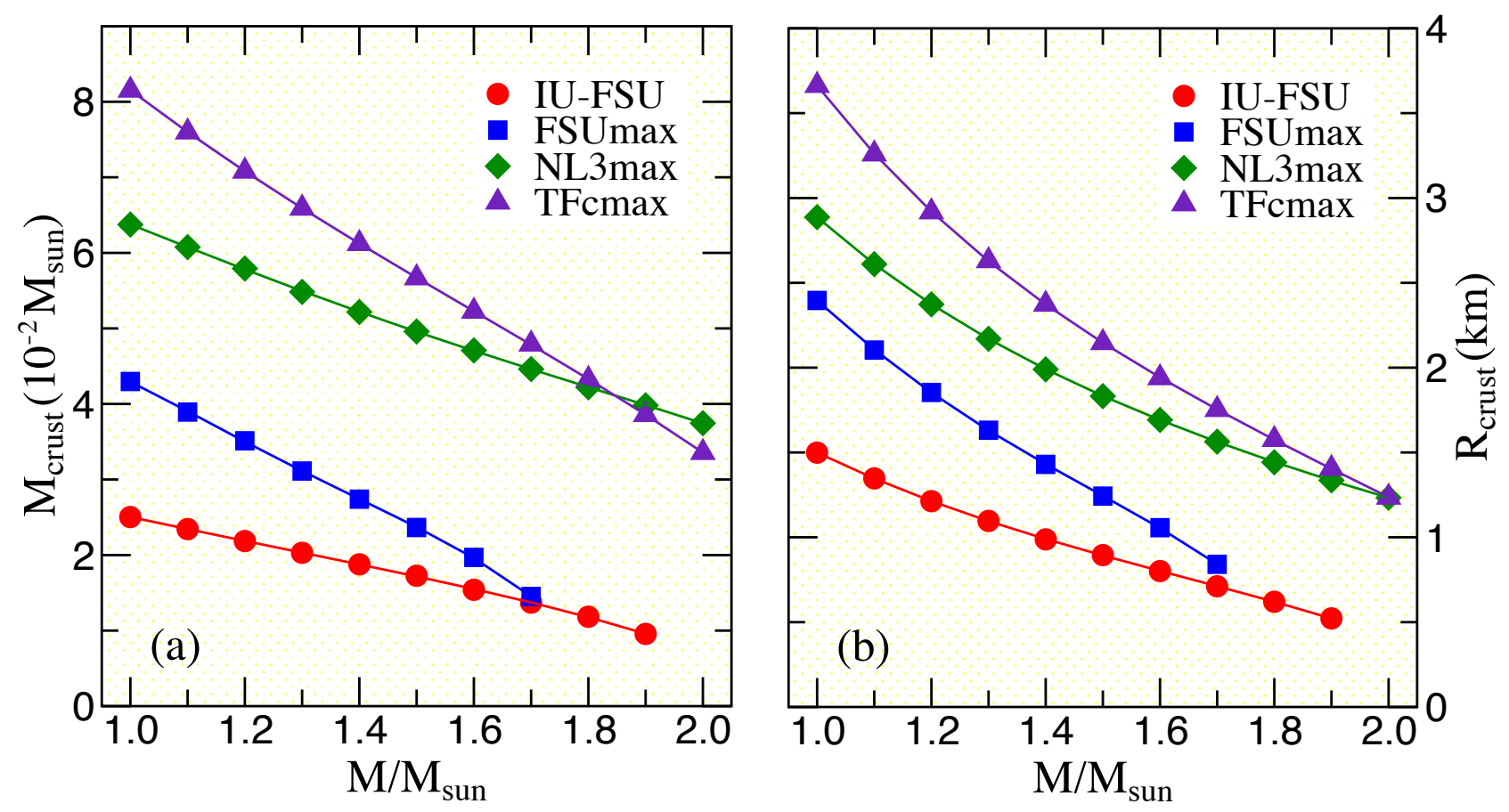

FIG. 3. (Color online) Crustal contributions to the total mass (a) and radius (b) as a function of neutron-star mass as predicted by the four models considered in the text. 


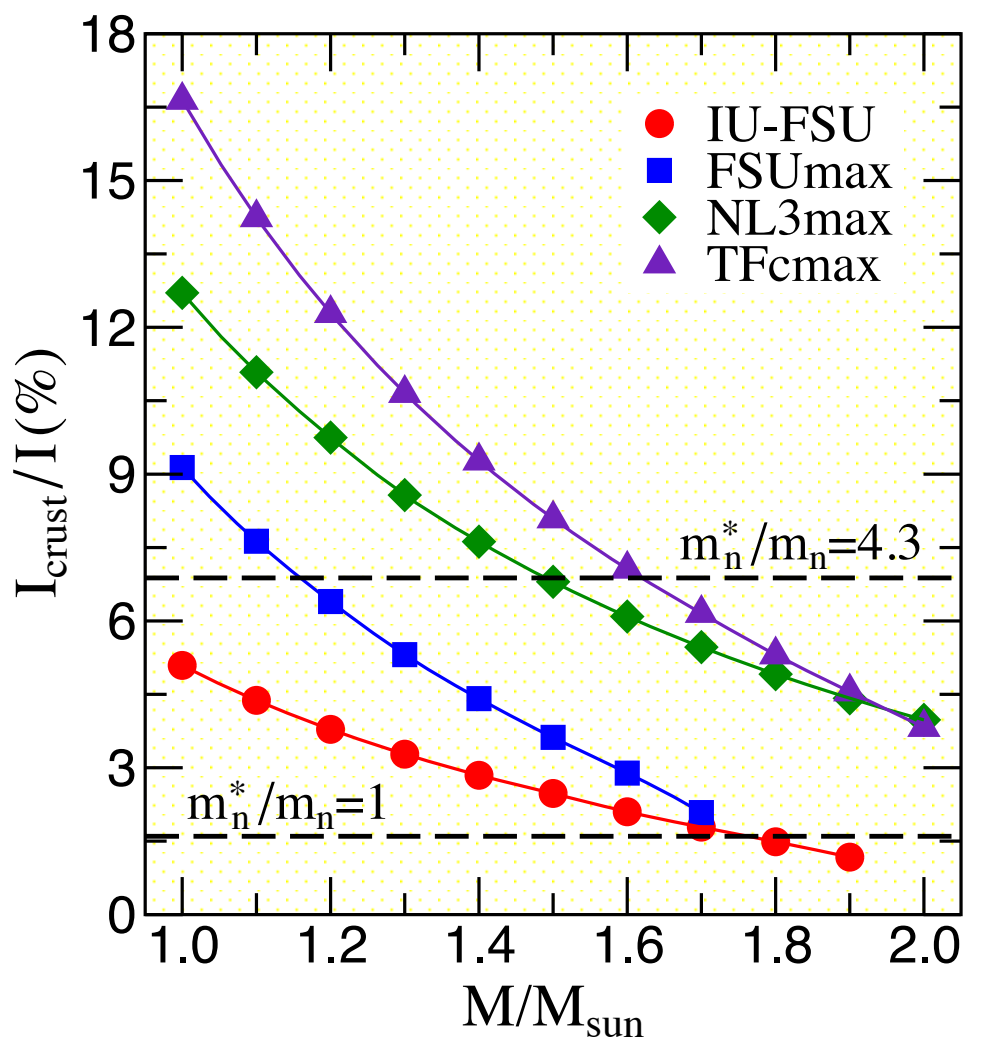

FIG. 4. (Color online) Fraction of the moment of inertia residing in the crust as a function of stellar mass for the four models considered in the text. The two horizontal lines represent observational constraints from the Vela glitches by assuming either no $\left(\left\langle m_{n\rangle}^{*} / m_{n}=1\right)\right.$ or significant $\left(\left\langle m_{n}^{*}\right\rangle / m_{n}=4.3\right)$ crustal entrainment.

We insist that although the analytic formulas displayed in Eqs.(8) and $(13)$ are highly insightful, the predictions made for all crustal properties displayed in Figs. 3 and 4 are exact - at least within the framework of the slow-rotation approximation. Our results indicate that even though the observation of large pulsar glitches in the presence of crustal entrainment provides a very stringent constraint on the EOS, some realistic theoretical models are consistent with such a constraint. Indeed, our findings suggest that one may account for the large Vela glitches even in the presence of significant crustal entrainment provided $M_{\text {vela }} \lesssim 1.6 M_{\odot}$. Note that a similar conclusion was reached in Ref. [20] from a study of neutron-star observations.

As mentioned earlier in Sec. IIB, the complexity of the inner stellar crust has hindered the construction of a detailed EOS and has made us rely on a polytropic approximation that interpolates between the solid outer crust and the uniform liquid core [57. Given the importance of crustal properties on our results, it is pertinent to examine the sensitivity of our results to our choice of $\gamma_{\mathrm{rFG}}=4 / 3$, which corresponds to the polytropic index of a non-interacting relativistic Fermi gas. To do so, we have examined in Table IV the sensitivity to the choice of polytropic index on the total stellar radius, total moment of inertia, crustal mass, crustal radius, and fractional moment of inertia for a $1.4 M_{\odot}$ neutron star. Note that for $\gamma_{\mathrm{rFG}}=4 / 3$, the results for the crustal properties are those quoted in TableIII. Although the crustal radius displays a strong sensitivity to the polytropic index - which correspondingly affects the total stellar radius - neither the crustal mass nor the total and fractional moments of inertia display dramatic changes. Indeed, Table IV displays a change of only $\lesssim 11 \%$ for the fractional moment of inertia. This result helps to further validate our main conclusion.

Before leaving this section, we would like to highlight some recent findings on stellar radii that appear to create significant tension with the constraints emerging from large pulsar glitches. Recently, Guillot et al. 64 were able to determine neutron-star radii by fitting the spectra of five quiescent low mass X-ray binaries (qLMXB) in globular clusters. By assuming that all neutron stars independent of their mass share a common radius $\left(R(M) \equiv R_{0}\right)$ they determined this common radius to be

$$
R_{0}=9.1_{-1.5}^{+1.3} \mathrm{~km}
$$




\begin{tabular}{|l||l|c|c|c|c|c|}
\hline Model & Polytrope Index & $R_{\text {tot }}(\mathrm{km})$ & $I_{\text {tot }}\left(10^{45} \mathrm{~g} \mathrm{~cm}^{2}\right)$ & $M_{\text {crust }}\left(M_{\odot}\right)$ & $R_{\text {crust }}(\mathrm{km})$ & $I_{\text {crust }} / I(\%)$ \\
\hline IU-FSU & $\gamma_{\text {min }}=1.000$ & 12.938 & 1.564 & 0.019 & 1.434 & 2.877 \\
& $\gamma_{\mathrm{rFG}}=1.333$ & 12.493 & 1.563 & 0.019 & 0.989 & 2.827 \\
& $\gamma_{\max }=2.420$ & 12.198 & 1.563 & 0.018 & 0.694 & 2.784 \\
\hline \hline FSUmax & $\gamma_{\text {min }}=1.000$ & 13.647 & 1.488 & 0.028 & 2.246 & 4.559 \\
& $\gamma_{\mathrm{rFG}}=1.333$ & 12.831 & 1.487 & 0.027 & 1.430 & 4.407 \\
& $\gamma_{\text {max }}=2.422$ & 12.300 & 1.486 & 0.027 & 0.900 & 4.287 \\
\hline \multirow{2}{*}{ NL3max } & $\gamma_{\min }=1.000$ & 15.610 & 1.866 & 0.054 & 3.290 & 7.970 \\
& $\gamma_{\mathrm{rFG}}=1.333$ & 14.313 & 1.862 & 0.052 & 1.990 & 7.619 \\
& $\gamma_{\max }=2.156$ & 13.591 & 1.860 & 0.051 & 1.267 & 7.380 \\
\hline \multirow{2}{*}{ TFcmax } & $\gamma_{\min }=1.000$ & 16.169 & 1.766 & 0.064 & 4.132 & 9.865 \\
& $\gamma_{\mathrm{rFG}}=1.333$ & 14.412 & 1.759 & 0.061 & 2.372 & 9.258 \\
& $\gamma_{\text {max }}=2.007$ & 13.542 & 1.755 & 0.060 & 1.499 & 8.917 \\
\hline
\end{tabular}

TABLE IV. Predictions for the total radius, total moment of inertia, crustal mass, crustal radius, and fractional moment of inertia for a $1.4 M_{\odot}$ neutron star as predicted by the various models used in the text.

Although several of the assumptions made in Ref. 64] suggest that other interpretations may be favored 65, Eq. (14) is highly intriguing as it suggests stellar radii significantly smaller than those predicted by many theoretical models 9 , 36 . Moreover, as one combines such a small radius with the observation of neutron stars with masses of $M \approx 2 M_{\odot}$ [42, 43], then most theoretical models appear to be ruled out. This is because, on the one hand, the pressure around twice nuclear-matter saturation density must be small enough to accommodate small stellar radii but, on the other hand, the pressure must stiffen significantly at high densities to support massive neutron stars.

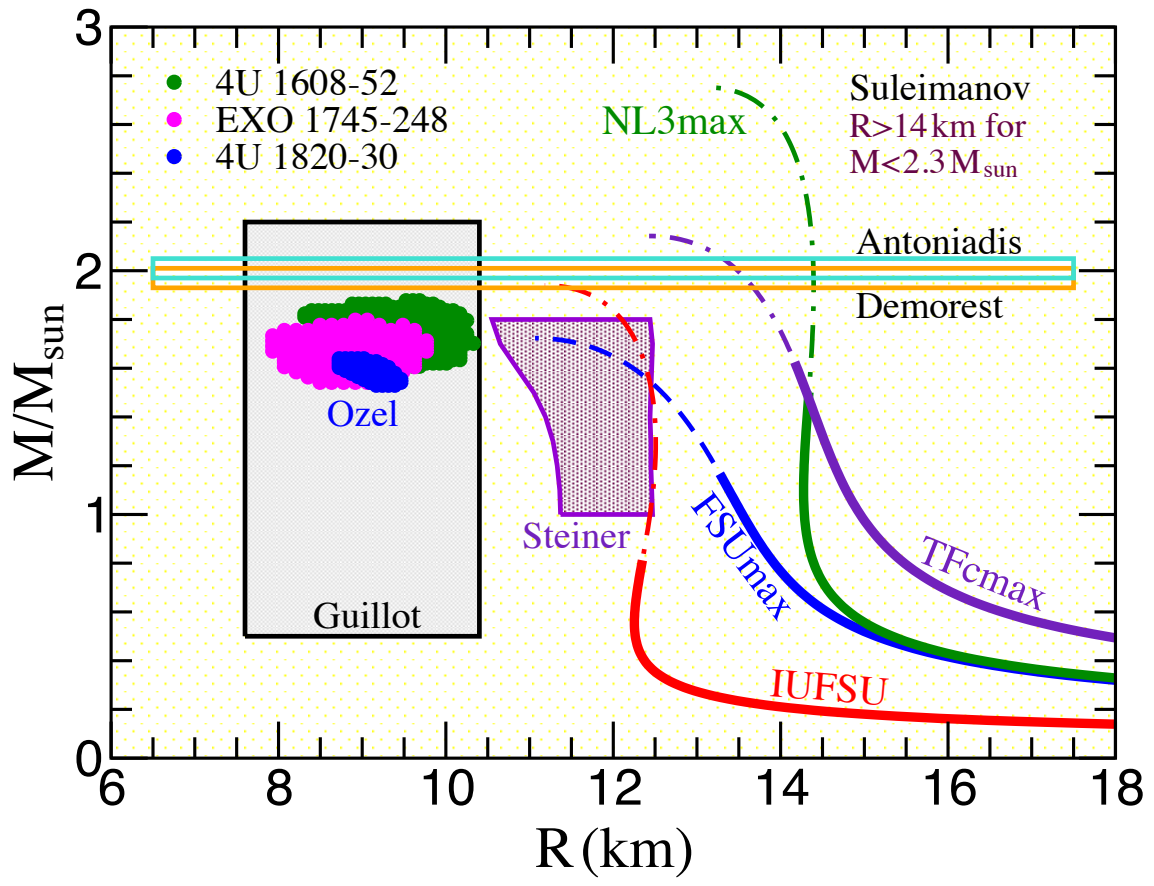

FIG. 5. (Color online) Mass-vs-Radius relationship predicted by the four models considered in the text. Also shown are observational constraints on stellar masses [42, 43] and radii [64, 66, 68, as explained in the text.

Such a situation is illustrated in Fig. 5 where the Mass-vs-Radius relationship is displayed for the four models considered in the text. The solid portion of each of these curves indicates the region that is consistent with the constraint from the large Vela glitches. That is, whereas IUFSU is compatible only with very small masses, TFcmax is consistent up to a mass of $1.6 M_{\odot}$. In addition to the "single-radius" finding of Guillot et al. 64, we display results obtained from the analysis of several X-ray bursters by Ozel et al. [66, Steiner et al. 67, and Suleimanov et al. 68]. However, given that systematic uncertainties in the analysis of X-ray bursters continue to hinder the reliable extraction 
of stellar radii 68, qLMXBs may provide, at least at present, a more reliable source for the determination of stellar radii. Also shown in the figure are the limits imposed by the measurement of two massive neutron stars [42, 43. It is evident from Fig.5 that none of our theoretical models are consistent with stellar radii significantly smaller than $12 \mathrm{~km}$. Indeed, to our knowledge very few_perhaps only one 69-EOS can simultaneously account for small radii and large masses. Note that we are unaware on whether such an EOS may be able to account for the large Vela glitches. In our case models such as TFcmax, that appear to account for both massive neutron stars and large pulsar glitches, predict stellar radii that are significantly larger than the common radius value of $R_{0}=9.1_{-1.5}^{+1.3} \mathrm{~km}$. Indeed, for a $1.4 M_{\odot}$ neutron star, TFcmax predicts a radius of $14.4 \mathrm{~km}$ - or $4 \mathrm{~km}$ larger than the suggested upper limit for $R_{0}$.

\section{CONCLUSIONS}

The large jumps observed in the spin frequency of some neutron stars like the Vela pulsar suggest the existence of an angular-momentum reservoir confined to the inner stellar crust. As such, large pulsar glitches have been used to constrain the EOS of neutron-rich matter by imposing limits on the fraction of the moment of inertia that must reside in the crust. Until recently, the large Vela glitches demanded that at least $1.6 \%$ of the stellar moment of inertia must be located in the crust. Although useful, many realistic EOS are compatible with such limit. However, recent studies seem to indicate that a significantly larger value is required once crustal entrainment is taken into account. Indeed, encoding the impact of crustal entrainment in a value of the effective neutron mass of $\left\langle m_{n}^{*}\right\rangle / m_{n}=4.3$, suggests that the limit on the fractional moment of inertia must be increased to almost $7 \%$. Unless the mass of the Vela pulsar is very small $\left(\lesssim 1 M_{\odot}\right)$ then a large number of theoretical models become incompatible with this much more stringent limit. This has lead to the assertion that the "crust is not enough".

The main goal of this work was to re-examine whether indeed the crust is not enough. Given that the crustal moment of inertia is highly sensitive to the transition pressure $P_{t}$ at the crust-core interface, we examined the predictions of a variety of relativistic mean-field models in the transition region. In particular, we found that certain bulk properties of the EOS at the crust-core interface are strongly correlated to the slope of the symmetry energy $L$. However, not the transition pressure. Indeed, $P_{t}$ increases monotonically for small $L$, reaches its maximum at some intermediate value, and ultimately decreases with increasing $L$. Given that in the class of RMF models used in this work one can easily tune the value of $L$, we searched for models with the largest transition pressure. By doing so, we were able to generate neutron stars with thick crusts and large crustal moments of inertia. Indeed, our results support the standard model of pulsar glitches — provided the Vela mass does not exceed 1.6 $M_{\odot}$. In particular, this requires values for the neutron-skin thickness of ${ }^{208} \mathrm{~Pb}$ of about $R_{\mathrm{skin}}^{208} \simeq(0.20-0.26) \mathrm{fm}$. This finding-which represents the central result of our work - offers yet another attractive connection between a laboratory measurement and an astrophysical observation.

We close with a few questions and comments on the impact of our findings on other neutron-star observables sensitive to crustal dynamics. First, rapidly rotating neutron stars with large asymmetries (such as mass quadrupoles) are efficient sources of gravitational waves. In particular, "large mountains" on rapidly rotating neutron stars may efficiently radiate gravitational waves provided that the breaking strain of the crust is large [70. Horowitz and Kadau have performed large-scale Molecular-Dynamics simulations that reveal a large breaking strain in support of large mountains in neutron stars 71. Such findings suggest that large mountains in rapidly rotating neutron stars may generate gravitational waves that may be detectable by the next generation of gravitational-wave observatories. Thus, one would like to understand the impact of the large crustal thicknesses found in our work $\left(e . g\right.$., $R_{\text {crust }} \simeq 2.4 \mathrm{~km}$ for a $1.4 M_{\odot}$ neutron star) on the breaking strain of the crust and ultimately on the emission of gravitational waves. Moreover, the size of the crust may also impact the cooling light curves of low-mass X-ray binaries during quiescence. Indeed, a thick stellar crust could increase the time scale for crustal cooling after extended periods of accretion; see Ref. [72] and references contained therein.

However, although the large crustal thicknesses raises interesting questions and accounts for large crustal fractions of the stellar moment of inertia, the moderate values of $L$ required to account for the large pulsar glitches are inconsistent with a recent analysis of quiescent low mass X-ray binaries that suggests very small stellar radii. In fact, the class of relativistic mean-field models employed in this work are unable to generate such small radii-regardless of whether one incorporates the pulsar-glitch constraint or not. Although not without controversy, we found instructive to take the small-radius result at face value and ask whether it is possible to account for both large pulsar glitches and small neutron stars. If crustal entrainment is as large as it has been suggested, then a moderate value of the neutronskin thickness of ${ }^{208} \mathrm{~Pb}$ is required. This prediction can be tested by performing parity violating electron scattering experiments at the Jefferson Laboratory. However, in order for neutron-star radii to be as small as suggested, a dramatic softening of the symmetry energy must develop by the time that the density reaches about 2 times nuclearmatter saturation density. Such a rapid softening is likely to involve a change in the structure of dense matter-likely due to a phase transition. In principle, the onset of such a phase transition may also be probed in the laboratory 
using energetic heavy-ion collisions of highly-asymmetric nuclei. Finally, the EOS must significantly stiffen at even higher densities in order to account for the observation of massive neutron stars. Such unique behavior of the EOS and its possible realization in neutron stars reaffirms the special bond between astrophysics and nuclear physics.

\section{ACKNOWLEDGMENTS}

This work was supported in part by grants DE-FG02-87ER40365 and DE-FD05-92ER40750 from the U.S. Department of Energy, by the National Aeronautics and Space Administration under grant NNX11AC41G issued through the Science Mission Directorate, and the National Science Foundation under grant PHY-1068022.

[1] C.M. Espinoza, A.G. Lyne, B.W. Stappers, and M. Kramer, Mon. Not. Roy. Astron. Soc. 414, 1679 (2011)

[2] "Jodrell Bank Centre for Astrophysics (glitch catalogue) http://www.jb.man.ac.uk/pulsar/glitches.html".

[3] P. Anderson and N. Itoh, Nature 256, 25 (1975)

[4] D. Pines and M. Alpar, Nature 316, 27 (1985).

[5] B. Link, R.I. Epstein, and J.M. Lattimer, Phys. Rev. Lett. 83, 3362 (1999).

[6] N. Chamel, Phys. Rev. Lett. 110, 011101 (2013)

[7] J. Hooker, W. Newton, and B.-A. Li, (2013), arXiv:1308.0031 [astro-ph.SR]

[8] G. Baym, C.J. Pethick, D. Pines, and N. Ruderman, Nature 224, 872 (1969).

[9] J.M. Lattimer and M. Prakash, Phys. Rept. 442, 109 (2007).

[10] F.J. Fattoyev and J. Piekarewicz, Phys. Rev. C82, 025810 (2010)

[11] N. Andersson, K. Glampedakis, W. Ho, and C. Espinoza, Phys. Rev. Lett. 109, 241103 (2012)

[12] N. Chamel, A. Fantina, J. Pearson, and S. Goriely, Phys. Rev. C84, 062802 (2011).

[13] C.J. Horowitz and J. Piekarewicz, Phys. Rev. Lett. 86, 5647 (2001).

[14] C. Ducoin, J. Margueron, and C. Providencia, Europhys. Lett. 91, 32001 (2010).

[15] A. Worley, P. G. Krastev, and B.-A. Li, (2008), arXiv:0801.1653 [astro-ph].

[16] J. Xu, L.-W. Chen, B.-A. Li, and H.-R. Ma, Phys. Rev. C79, 035802 (2009)

[17] J. Xu, L.-W. Chen, B.-A. Li, and H.-R. Ma, Astrophys. J. 697, 1549 (2009)

[18] C. Moustakidis, T. Niksic, G. Lalazissis, D. Vretenar, and P. Ring, Phys. Rev. C81, 065803 (2010).

[19] N. Paar, C.C. Moustakidis, T. Marketin, D. Vretenar, and G. Lalazissis, (2014), arXiv:1403.7574 [nucl-th].

[20] A.W. Steiner, S. Gandolfi, F.J. Fattoyev, and W.G. Newton, (2014), arXiv:1403.7546 [nucl-th].

[21] B. A. Brown, Phys. Rev. Lett. 85, 5296 (2000).

[22] R.J. Furnstahl, Nucl. Phys. A706, 85 (2002).

[23] M. Centelles, X. Roca-Maza, X. Viñas, and M. Warda, Phys. Rev. Lett. 102, 122502 (2009)

[24] X. Roca-Maza, M. Centelles, X. Viñas, and M. Warda, Phys. Rev. Lett. 106, 252501 (2011)

[25] S. Abrahamyan, Z. Ahmed, H. Albataineh, K. Aniol, D. Armstrong, et al., Phys.Rev.Lett. 108, 112502 (2012)

[26] C.J. Horowitz, Z. Ahmed, C. Jen, A. Rakhman, P. Souder, et al., Phys. Rev. C85, 032501 (2012)

[27] K. Paschke, K. Kumar, R. Michaels, P. A. Souder, and G. M. Urciuoli, (2012).

[28] J.B. Hartle, Astrophys. J. 150, 1005 (1967).

[29] J.B. Hartle and K.S. Thorne, Astrophys. J. 153, 807 (1968).

[30] J.B. Hartle, Astrophys. Space Sci. 24, 385 (1973)

[31] F. Weber, "Pulsars as astrophysical laboratories for nuclear and particle physics," (Institute of Physics Publishing, Bristol, UK, 1999).

[32] N.K. Glendenning, "Compact stars," (Springer-Verlag New York, 2000).

[33] C.P. Lorenz, D.G. Ravenhall, and C.J. Pethick, Phys. Rev. Lett. 70, 379 (1993)

[34] D.G. Ravenhall and C.J. Pethick, Astrophys. J. 424, 846 (1994)

[35] J.M. Lattimer and M. Prakash, Astrophys. J. 550, 426 (2001).

[36] C.J. Horowitz, E. Brown, Y. Kim, W. Lynch, R. Michaels, et al., (2014), arXiv:1401.5839 [nucl-th].

[37] J.D. Walecka, Annals Phys. 83, 491 (1974).

[38] B.D. Serot and J.D. Walecka, Adv. Nucl. Phys. 16, 1 (1986).

[39] B.D. Serot and J.D. Walecka, Int. J. Mod. Phys. E6, 515 (1997).

[40] J. Boguta and A.R. Bodmer, Nucl. Phys. A292, 413 (1977).

[41] H. Mueller and B.D. Serot, Nucl. Phys. A606, 508 (1996).

[42] P. Demorest, T. Pennucci, S. Ransom, M. Roberts, and J. Hessels, Nature 467, 1081 (2010).

[43] J. Antoniadis, P.C. Freire, N. Wex, T.M. Tauris, R.S. Lynch, et al., Science 340, 6131 (2013)

[44] C.J. Horowitz and J. Piekarewicz, Phys. Rev. C64, 062802 (2001).

[45] B.G. Todd and J. Piekarewicz, Phys. Rev. C67, 044317 (2003).

[46] F.J. Fattoyev and J. Piekarewicz, Phys. Rev. Lett. 111, 162501 (2013)

[47] G. Baym, C. Pethick, and P. Sutherland, Astrophys. J. 170, 299 (1971).

[48] X. Roca-Maza and J. Piekarewicz, Phys. Rev. C78, 025807 (2008) 
[49] D.G. Ravenhall, C.J. Pethick, and J.R. Wilson, Phys. Rev. Lett. 50, 2066 (1983).

[50] M. Hashimoto, H. Seki, and M. Yamada, Prog. Theor. Phys. 71, 320 (1984).

[51] G. Watanabe, K. Sato, K. Yasuoka, and T. Ebisuzaki Phys. Rev. C68, 035806 (2003).

[52] G. Watanabe, T. Maruyama, K. Sato, K. Yasuoka, and T. Ebisuzaki, Phys. Rev. Lett. 94, 031101 (2005).

[53] C.J. Horowitz, M.A. Perez-Garcia, and J. Piekarewicz, Phys. Rev. C69, 045804 (2004).

[54] C.J. Horowitz, M.A. Perez-Garcia, J. Carriere, D.K. Berry, and J. Piekarewicz, Phys. Rev. C70, 065806 (2004).

[55] C.J. Horowitz, M.A. Perez-Garcia, D.K. Berry, and J. Piekarewicz, Phys. Rev. C72, 035801 (2005).

[56] A. Schneider, C.J. Horowitz, J. Hughto, and D.K. Berry, Phys. Rev. C88, 065807 (2013).

[57] J. Carriere, C.J. Horowitz, and J. Piekarewicz, Astrophys. J. 593, 463 (2003).

[58] B.G. Todd-Rutel and J. Piekarewicz, Phys. Rev. Lett 95, 122501 (2005).

[59] G.A. Lalazissis, J. Konig, and P. Ring, Phys. Rev. C55, 540 (1997).

[60] G.A. Lalazissis, S. Raman, and P. Ring, At. Data Nucl. Data Tables 71, 1 (1999).

[61] F.J. Fattoyev, C.J. Horowitz, J. Piekarewicz, and G. Shen, Phys. Rev. C82, 055803 (2010).

[62] J. Piekarewicz and M. Centelles, Phys. Rev. C79, 054311 (2009).

[63] J. Piekarewicz, Eur. Phys. J. A 50, 25 (2013).

[64] S. Guillot, M. Servillat, N.A. Webb, and R.E. Rutledge, Astrophys. J. 772, 7 (2013).

[65] J.M. Lattimer and A.W. Steiner, (2013), arXiv:1305.3242 [astro-ph.HE]

[66] F. Ozel, G. Baym, and T. Guver, Phys. Rev. D82, 101301 (2010).

[67] A.W. Steiner, J.M. Lattimer, and E.F. Brown, Astrophys. J. 722, 33 (2010)

[68] V. Suleimanov, J. Poutanen, M. Revnivtsev, and K. Werner, Astrophys. J. 742, 122 (2011)

[69] R. B. Wiringa, V. Fiks, and A. Fabrocini, Phys. Rev. C38, 1010 (1988)

[70] N. Andersson, V. Ferrari, D. Jones, K. Kokkotas, B. Krishnan, et al., Gen. Rel. Grav. 43, 409 (2011)

[71] C.J. Horowitz and K. Kadau, Phys. Rev. Lett. 102, 191102 (2009)

[72] E.F. Brown and A. Cumming, Astrophys. J. 698, 1020 (2009). 